\title{
Follow-Up MRI for Small Brain AVMs Treated by Radiosurgery: Is Gadolinium Really Necessary?
}

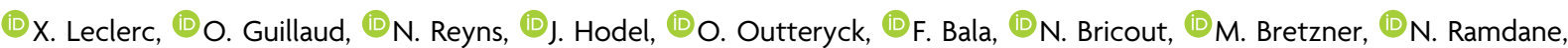 \\ (D).-P. Pruvo, 는. Hacein-Bey, and $\mathbb{1}^{\mathrm{G}} \mathrm{G}$. Kuchcinski
}

\begin{abstract}
BACKGROUND AND PURPOSE: Follow-up MR imaging of brain AVMs currently relies on contrast-enhanced sequences. Noncontrast techniques, including arterial spin-labeling and TOF, may have value in detecting a residual nidus after radiosurgery. The aim of this study was to compare noncontrast with contrast-enhanced MR imaging for the differentiation of residual-versus-obliterated brain AVMs in radiosurgically treated patients.
\end{abstract}

MATERIALS AND METHODS: Twenty-eight consecutive patients with small brain AVMs $(<20 \mathrm{~mm})$ treated by radiosurgery were followed with the same MR imaging protocol. Three neuroradiologists, blinded to the results, independently reviewed the following: 1) postcontrast images alone (4D contrast-enhanced MRA and postcontrast $3 \mathrm{D} T 1$ gradient recalled-echo), 2) arterial spin-labeling and TOF images alone, and 3) all MR images combined. The primary end point was the detection of residual brain AVMs using a 5point scale, with DSA as the reference standard.

RESULTS: The highest interobserver agreement was for arterial spin-labeling/TOF $(\kappa=0.81$; 95\% confidence interval, 0.66-0.93). Regarding brain AVM detection, arterial spin-labeling/TOF had higher sensitivity (sensitivity, 85\%; specificity, 100\%; 95\% Cl, 62-97) than contrast-enhanced MR imaging (sensitivity, 55\%; specificity, 100\%; $95 \% \mathrm{Cl}, 27-73$ ) and all MR images combined (sensitivity, $75 \%$; specificity, 100\%; $95 \% \mathrm{Cl}$, 51-91) $(P=.008)$. All nidus obliterations on DSA were detected on MR imaging. In 6 patients, a residual brain AVM present on DSA was only detected with arterial spin-labeling/TOF, including 3 based solely on arterial spin-labeling images.

CONCLUSIONS: In this study of radiosurgically treated patients with small brain AVMs, arterial spin-labeling/TOF was found to be superior to gadolinium-enhanced MR imaging in detecting residual AVMs.

ABBREVIATIONS: ASL = arterial spin-labeling; AVS = arteriovenous shunting; BAVM = brain AVM; GRE = gradient recalled-echo; PLD = post label delay

B rain AVMs (BAVMs) are characterized by abnormal communications between the arterial and venous circulations without a normal interposed capillary bed in the form of multiple feeding arteries, an abnormal capillary network (nidus), and enlarged draining veins. The main risk of BAVMs is hemorrhage, which occurs in $2 \%-4 \%$ of patients per year and may

Received September 26, 2019; accepted after revision December 17

From the Departments of Neuroradiology (X.L., O.G., O.O., F.B., N.B., M.B., J.-P.P., G.K.), Neurosurgery (N. Reyns), Neurology (O.O.), Centre Hospitalier Universitaire Lille, Lille, France; Inserm U1171, Degenerative and Vascular Cognitive Disorders (X.L., O.O., J.-P.P., G.K.), University of Lille, Lille, France; Inserm U1189-ONCO-THAI-Image Assisted Laser Therapy for Oncology (N. Reyns), University of Lille, Lille, France; Department of Neuroradiology (J.H.), Hôpital Henri Mondor, Créteil, France; EA 2694-Public Health: Epidemiology and Quality of Care (N. Ramdane), University of Lille, Centre Hospitalier Universitaire Lille, Lille, France; and Neuroradiology,

Radiology Department (L.H.-B.), University of California Davis School of Medicine, Sacramento, California.

Please address correspondence to Xavier Leclerc, MD, Neuroradiology Department, Hôpital Roger Salengro, Rue Emile Laine, 59037 Lille, France; e-mail: xavier.leclerc@chru-lille.fr

http://dx.doi.org/10.3174/ajnr.A6404 lead to disability or even death. ${ }^{1-3}$ The treatment of BAVMs depends on the clinical presentation (including prior hemorrhage) and size and location of the nidus ${ }^{4}$ and may combine various modalities, including surgery, embolization, and/or radiosurgery. Radiosurgery, which allows progressive vascular obliteration for up to 5 years by radiation-induced obliterative endarteritis, is most often used for small BAVMs in deep or eloquent locations. ${ }^{5,6}$

DSA is the reference standard to evaluate treatment efficacy at follow-up owing to high spatial resolution and superior temporal resolution relative to MR imaging, allowing differentiation of arterial and venous angiographic phases. ${ }^{7}$ However, DSA is an invasive test, which may result in neurologic and vascular complications. ${ }^{8}$ MR imaging, which is noninvasive, is increasingly used to evaluate treatment effectiveness after radiosurgery. At our institution, time-resolved (4D) contrast MRA and postcontrast 3D T1 gradient recalled-echo (GRE) sequences are routinely used to assess gradual nidus obliteration after radiosurgery. However, 
the sensitivity and specificity of contrast-enhanced MR images to detect a small nidus remain low, mainly because of inherent limited spatial resolution. ${ }^{9,10}$ Furthermore, serial injections of gadolinium-based contrast agent across time may prove harmful, for various reasons, including deposition in deep gray nuclei. ${ }^{11,12}$

Studies have shown the usefulness of 3D-TOF and arterial spin-labeling (ASL) for the detection of BAVMs. 3D-TOF is sensitive for detecting BAVMs because faster blood flow within the BAVM causes hypersignal from the inflow effect. However, the detection of small BAVMs can remain challenging. ${ }^{13}$ On the other hand, ASL, which relies on arterial flow proton labeling at the cervical level, allows measuring cerebral blood flow. Following a short (1500-2000 ms) postlabel delay (PLD), brain images are acquired at the time magnetically labeled protons flow into the capillary bed. ${ }^{14,15}$ Labeled protons do not normally reach venous structures, which then appear hypointense due to T1 decay during capillary transit. However, Wolf et $\mathrm{al}^{16}{ }^{16}$ in 2008 , demonstrated the presence of hyperintense signal in the venous structures on ASL maps in patients with BAVM, and Le et al, ${ }^{17}$ in 2012, showed that venous ASL signal intensity was the strongest predictor of arteriovenous shunting (AVS) on DSA.

We hypothesized that ASL/TOF is as sensitive as contrastenhanced MR imaging in detecting either nidus obliteration or residual shunting in patients treated with radiosurgery.

In this study of a cohort of patients with small BAVMs $(<20 \mathrm{~mm})$ treated with gamma knife radiosurgery and followed by DSA, we aimed to evaluate the comparative performance levels of noncontrast MR imaging, including ASL and 3D-TOF, and contrast-enhanced MR imaging, including 4D contrast MRA and postcontrast 3D T1 GRE, in assessing nidus obliteration or a residual BAVM.

\section{MATERIALS AND METHODS \\ Patient Population}

This retrospective study was approved by our institutional review board of Lille University. We selected patients with a high likelihood of nidus obliteration or small residual shunting for whom MR imaging is highly challenging. Inclusion criteria were as follows:

- MRI studies obtained on a 3T MR imaging scanner

- Nidus size (maximum diameter) of $\leq 20 \mathrm{~mm}$ after radiosurgery

- Last treatment procedure by radiosurgery alone or by embolization and radiosurgery at least 2 years before DSA

- Time interval between DSA and MRI of $\leq 2$ months

- No BAVM treatment between DSA and MRI.

From February 2017 to March 2019, forty-seven consecutive patients with small BAVMs underwent follow-up imaging with DSA and 3T MR imaging, including TOF and ASL, after gamma knife radiosurgery. Of those 47 patients, 19 were excluded from this study for the following reasons: a BAVM size of $>20 \mathrm{~mm}$ in 9 patients, early DSA control after radiosurgery ( 6 months) in 6 patients, and a long delay between DSA and MR imaging in 4 patients.
Twenty-eight consecutive patients (10 women/18 men; mean age, 41 years; range, 17-65 years) remained in the study. In this cohort, BAVM presentation was intracranial hemorrhage $(n=20)$, seizures $(n=5)$, and incidental discovery $(n=3)$. Radiosurgery alone was used in 13 patients, and embolization followed by radiosurgery, in 15. DSA was performed in all patients after brain MR imaging to confirm BAVM obliteration or to evaluate the size of the residual nidus. The mean time interval between radiosurgery and MR imaging was 4.2 years (range, 2-10 years), and the mean time interval between MR imaging and DSA was 10 days (range, 1-45 days).

\section{Imaging Methods}

DSA was performed on a flat panel biplane system (AlluraClarity; Philips Healthcare, Best, the Netherlands). Selective bilateral carotid and vertebral angiograms with at least 2 orthogonal views (anteroposterior and lateral) and 3D rotational angiography acquired at 3 frames per second with a $1024 \times 1024$ matrix size and a $20-\mathrm{cm}$ FOV were obtained. Superselective feeding artery angiography with focused angiographic views was consistently performed if there was uncertainty as to the presence of a residual nidus.

MR imaging examinations were performed on a $3 \mathrm{~T}$ scanner (Achieva; Philips Healthcare) with a 32-channel head coil. Imaging protocol included the following sequences: DWI ( $b=1000 \mathrm{~s} / \mathrm{mm}^{2}$; TR/TE, 2827/67 ms), axial FLAIR (TR/TE/TI, $11,000 / 125 / 2800 \mathrm{~ms}$ ), axial T2* (TR/TE, $890 / 16 \mathrm{~ms}$ ) or SWI (TR/ TE, 28/5.6 ms), axial spin-echo T1 (TR/TE, 531/10 ms), 3D-TOF (TR/TE, 25/3.5 ms; FOV, $19 \mathrm{~cm}$; matrix, $472 \times 270$; section thickness, $1.2 \mathrm{~mm}$; scan time, 5 minutes 39 seconds), 2D pseudocontinuous ASL (TR/TE, 4297/14 ms; FOV, 24 cm; labeling period, $1650 \mathrm{~ms}$ followed by a PLD of $2000 \mathrm{~ms}$; section thickness, $5 \mathrm{~mm}$; scan time, 4 minutes 55 seconds), 4D contrast MRA (TR/TE, 3/ $1.1 \mathrm{~ms}$; FOV, $24 \mathrm{~cm}$; matrix, $240 \times 240$; temporal resolution, 1.1 second per volume; scan time, 1 minutes 4 seconds), halfbrain coverage ipsilateral to the BAVM side, and postcontrast $3 \mathrm{D}$ T1 GRE (TR/TE, 7.7/3.7 ms; FOV, $24 \mathrm{~cm}$; matrix, $240 \times 240$; scan time 3 minutes 43 seconds). Intravenous gadoterate meglumine (Dotarem; Guerbet, Aulnay-sous-Bois, France) was administered at a concentration of $0.1 \mathrm{mmol} / \mathrm{kg}$ and a rate of $1.5 \mathrm{~mL} / \mathrm{s}$.

\section{Imaging Analysis}

DSA examinations were interpreted conjointly by 2 interventional neuroradiologists (N.B., X.L.), each with $>8$ years of experience. The presence of a residual nidus or early venous drainage was recorded. BAVM nidus maximum diameter, location, and drainage type were recorded. Evidence of a draining vein on the arterial phase of angiograms without a measurable nidus was defined as a residual AVS.

MR images were de-identified and randomly interspersed. Three reviewers, a diagnostic neuroradiologist with 10 years of experience (G.K.), a senior interventional neuroradiologist (F.B.) with 6 years of experience, and a junior neuroradiologist (O.O.) with 3 years of experience, blinded to the clinical data and MR imaging/DSA correlations, independently reviewed MR imaging examinations in random order. MR images were reviewed at 3 separate sessions, each separated by a month. At each review 
Table 1: Patient characteristics and imaging findings after gamma knife radiosurgery

\begin{tabular}{|c|c|c|c|c|c|c|c|c|}
\hline \multicolumn{3}{|c|}{ Patients } & \multicolumn{3}{|c|}{ DSA } & \multicolumn{3}{|c|}{ MRI } \\
\hline No. & Age (yr) & Sex & Presentation & Location & Drainage & Findings & Contrast MRI & ASL/TOF \\
\hline 1 & 65 & $M$ & Hemorrhage & L temporal & Superficial & AVS & - & + \\
\hline 2 & 23 & $\mathrm{~F}$ & Hemorrhage & $\mathrm{L}$ thalamus & Deep & AVS & - & + \\
\hline 3 & 17 & M & Hemorrhage & L frontal & Mixed & Nidus 13 mm & + & + \\
\hline 4 & 36 & M & Hemorrhage & $\mathrm{R}$ frontal & Superficial & AVS & - & - \\
\hline 5 & 64 & M & Hemorrhage & L cerebellar & Deep & $\mathrm{O}$ & - & - \\
\hline 6 & 47 & $M$ & Hemorrhage & R frontal & Superficial & AVS & - & + \\
\hline 7 & 45 & $\mathrm{~F}$ & Hemorrhage & L parietal & Mixed & Nidus $9 \mathrm{~mm}$ & + & + \\
\hline 8 & 35 & M & Hemorrhage & $\mathrm{R}$ frontal & Deep & Nidus 14 mm & + & + \\
\hline 9 & 22 & $\mathrm{~F}$ & Incidental & L temporal & Superficial & AVS & - & - \\
\hline 10 & 47 & $M$ & Hemorrhage & R occipital & Deep & O & - & - \\
\hline 11 & 27 & M & Hemorrhage & L occipital & Superficial & AVS & - & + \\
\hline 12 & 41 & $M$ & Seizure & L frontal & Deep & $\mathrm{O}$ & - & - \\
\hline 13 & 52 & $\mathrm{~F}$ & Hemorrhage & L temporal & Deep & Nidus 10 mm & + & + \\
\hline 14 & 37 & $\mathrm{~F}$ & Incidental & L frontal & Superficial & O & - & - \\
\hline 15 & 65 & $\mathrm{~F}$ & Hemorrhage & L temporal & Superficial & AVS & - & - \\
\hline 16 & 30 & $\mathrm{~F}$ & Hemorrhage & R parietal & Superficial & Nidus 18 mm & + & + \\
\hline 17 & 40 & $M$ & Seizure & R frontal & Superficial & Nidus $20 \mathrm{~mm}$ & + & + \\
\hline 18 & 31 & $M$ & Seizure & L parietal & Superficial & Nidus 17 mm & + & + \\
\hline 19 & 33 & $\mathrm{~F}$ & Hemorrhage & L parietal & Deep & Nidus 12 mm & + & + \\
\hline 20 & 26 & $\mathrm{~F}$ & Hemorrhage & L temporal & Deep & Nidus $8 \mathrm{~mm}$ & + & + \\
\hline 21 & 60 & $M$ & Hemorrhage & L parietal & Mixed & Nidus $5 \mathrm{~mm}$ & - & + \\
\hline 22 & 37 & M & Hemorrhage & $\mathrm{R}$ parietal & Superficial & $\mathrm{O}$ & - & - \\
\hline 23 & 42 & $\mathrm{~F}$ & Hemorrhage & R parietal & Deep & $\mathrm{O}$ & - & - \\
\hline 24 & 50 & $M$ & Incidental & $\mathrm{R}$ frontal & Superficial & O & - & - \\
\hline 25 & 23 & $M$ & Seizure & L temporal & Superficial & Nidus $11 \mathrm{~mm}$ & + & + \\
\hline 26 & 56 & M & Hemorrhage & R frontal & Deep & 0 & - & - \\
\hline 27 & 53 & $M$ & Hemorrhage & R cerebellar & Mixed & AVS & - & + \\
\hline 28 & 54 & $M$ & Seizure & R frontal & Mixed & Nidus 15 mm & + & + \\
\hline
\end{tabular}

Note:-+, residual brain arteriovenous malformation; -, nidus obliteration; L, left; R, right; O, occlusion.

session, attention was paid to the presence of hemorrhage, edema, abnormal vessels, and embolization material on FLAIR, T2*, and/or SWI sequences.

At the first review session, only contrast-enhanced MR images (4D contrast MRA and postcontrast 3D T1 GRE) were added to FLAIR and $\mathrm{T}^{*} / \mathrm{SWI}$. The presence of a nidus, abnormal serpiginous vessels, early venous drainage, and radiation-induced contrast enhancement was recorded. Contrast enhancement was interpreted as radiation-related injury when it showed nodular or heterogeneous contrast enhancement on postcontrast 3D T1 GRE at the site of the radiated nidus without an early draining vein on $4 \mathrm{D}$ contrast MRA. At the second review session, only non-contrast-enhanced MR images (3D TOF, ASL FLAIR, and $\mathrm{T} 2 * / \mathrm{SWI})$ were assessed. The presence of hypersignal at the site of the nidus and/or within neighboring venous structures was noted. At the time of the third and final review session, all MR images were available for interpretation.

After each case, readers used a 5-point scale to rate the likelihood of a residual AVM without distinction between the nidus and AVS: 1) very unlikely, 2) unlikely, 3) uncertain, 4) likely, and 5) very likely. The 2 noncontrast (ASL and TOF) and the 2 contrast-enhanced (4D contrast-MRA and postcontrast 3D T1 GRE) techniques were interpreted conjointly but rated separately. Cases resulting in disagreement among readers were then jointly reviewed by the 3 readers to reach a consensus. For the statistical analysis, the 5-point scale was reduced to a 3-point scale as follows: negative (1 and 2), uncertain (3), and positive (4 and 5) for the likelihood of a residual AVM.

\section{Statistical Analysis}

Data were analyzed using the SAS software package, Release 9.4 (SAS Institute, Cary, North Carolina). Results were presented as numbers and percentages for categoric variables. Sensitivity, specificity, and predictive values were calculated for the 3 readers separately, the consensus results, and the 3 sessions using DSA as the reference standard. Sensitivity and specificity of the consensus data were compared using the McNemar test. Interobserver agreement on the diagnosis for each sequence was assessed using a weighted $\kappa$ coefficient or a Cohen $\kappa$ coefficient. $^{18}$ The Krippendorf $\alpha$ coefficient was also calculated to measure the agreement among the different observers as a whole. This was interpreted as follows ${ }^{19}$ : poor agreement for values $<0.45$, fair to good agreement for values between 0.45 and 0.75 , and excellent agreement for values $>0.75 . \kappa$ coefficients within $95 \%$ confidence intervals were also calculated. Statistical testing was conducted as the 2-tailed $\alpha$ level of .05.

\section{RESULTS}

\section{DSA Findings}

Patient characteristics and imaging findings after radiosurgery are presented in Table 1 . Of the 28 BAVMs radiosurgically treated, follow-up DSA revealed that 8 were obliterated, 12 had a residual nidus (mean size, $12.5 \mathrm{~mm}$; range, $5-20 \mathrm{~mm}$ ), and $8 \mathrm{had}$ a residual AVS (nonmeasurable nidus). Venous drainage was superficial in 13 patients, deep in 10 patients, and mixed in 5 patients. 


\section{Interobserver Agreement}

Interobserver agreement data ( $\kappa$ value, 95\% CI) are described in Table 2. The overall agreement was highest at the second session (ASL/TOF) with $\kappa=0.81$ (95\% CI, 0.66-0.93). There were 3 disagreements with ASL: In 2 cases, hypersignal at the site of the nidus was judged too faint to be related to a residual BAVM by reader 2 in one case and by readers 1 and 3 in another case. The third disagreement concerned faint hypersignal considered to represent a residual BAVM by readers 2 and 3, but judged artifactual by reader 1 . In 1 case, contrast enhancement on 3D T1 GRE at the site of the nidus was read as a residual BAVM by readers 2 and 3 and as radiation-induced contrast enhancement by reader 1 .

\section{Comparison of All MR Imaging Sequences and DSA}

The sensitivity, specificity, positive predictive value, and negative predictive value of MR imaging (all sequences combined) for the detection of residual AVM were respectively 75\% (95\% CI, 5191), 100\% (95\% CI, 63-100), 100\% (95\% CI, 78-100), and 61.5\% (95\% CI, 32-86). Individual results are presented in Table 3. In 2 patients, all 3 reviewers reported a BAVM obliteration on MR imaging, whereas DSA demonstrated residual AVS. Of the 6 patients with MR images interpreted as uncertain, DSA revealed residual AVS in 3 patients and nidus obliteration in 3 patients. Fig 1 shows an illustrative example of a residual BAVM demonstrated on both contrastenhanced MR images and ASL/TOF.

\section{Contrast-Enhanced MR Imaging Sequences for the Detection of Residual BAVM}

Sensitivity, specificity, positive predictive value, and negative predictive value of contrast MR imaging for the detection of residual BAVMs were respectively $55 \%$ (95\% CI, 27-73), $100 \%$ (95\% CI, 63-100), 100\% (95\%

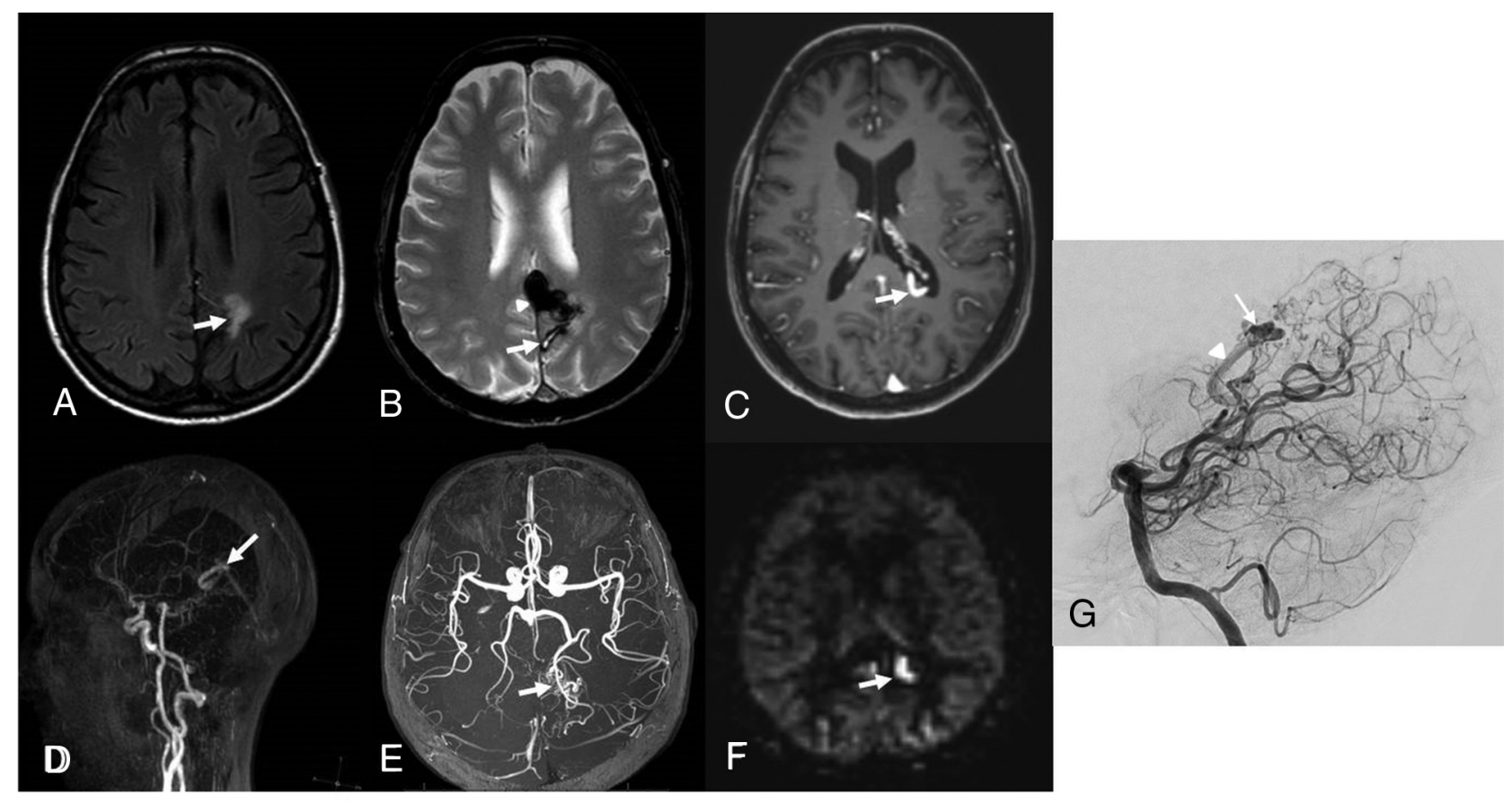

FIG 1. Patient 19. Follow-up MR imaging 2 years after embolization and radiosurgery for a ruptured left parietal brain AVM. A, Axial FLAIR shows hyperintense signal in the left parietal region (arrow) due to radiation injury. $B$, Axial T2* shows mixed signal intensity related to prior hemorrhage (arrow) posterior to hypointense embolization material (arrowhead). C, Postcontrast 3D T1 GRE axial image shows an enhanced tubular draining vein of a residual BAVM (arrow). D, Sagittal view from 4D contrast MRA with MIP reconstruction shows an early enhanced draining vein on the arterial phase of the angiogram (arrow). E, 3D-TOF with MIP reconstruction shows a residual BAVM (arrow). F, ASL image shows a focal hyperintense signal in the left lateral part of the cingulate gyrus (arrow). G, DSA, lateral view from a vertebral angiogram, confirms a residual nidus (arrow) with deep venous drainage (arrowhead). 
CI, 69-100), and 47\% (95\% CI, 21-69). Individual results are described in Table 4. There were 9 false-negatives of a DSA-proved residual nidus or AVS, which were read as BAVM obliteration on contrast-enhanced MR images. In 5 patients, postcontrast 3D T1 GRE images showed radiation-induced contrast enhancement at the site of the radiosurgically treated nidus, including nodular enhancement (Fig 2) in 3 and linear-like heterogeneous enhancement in 2 (Fig 3). Of the 5 patients, 4 were given a very unlikely or unlikely score ( $1 / 5$ or $2 / 5$, respectively), and 1 , an uncertain score (3/5). In the 5 patients with radiation-induced changes, 4D MRA findings were interpreted as negative, whereas DSA showed a residual BAVM in 3 and nidus obliteration in 2. In 4 other patients, 4D-MRA findings were considered negative, whereas a residual BAVM was demonstrated on postcontrast 3D T1 GRE.

\section{ASL/TOF for the Detection of Residual BAVMs}

The sensitivity, specificity, positive predictive value, and negative predictive value of ASL/TOF for the detection of residual BAVMs

Table 4: Sensitivity, specificity, PPV, and NPV of the first sitting (only FLAIR, T2*, and contrast-enhanced MR images)

\begin{tabular}{lcccc}
\multicolumn{1}{c}{ Readers } & Sensitivity (95\% CI) & Specificity (95\% CI) & PPV (95\% CI) & NPV (95\% CI) \\
\hline 1 & $55 \%(31-77)$ & $100 \%(63-100)$ & $100 \%(71-100)$ & $47 \%(23-72)$ \\
2 & $55 \%(31-77)$ & $100 \%(63-100)$ & $100 \%(71-100)$ & $47 \%(23-72)$ \\
3 & $45 \%(23-68)$ & $100 \%(63-100)$ & $100 \%(66-100)$ & $42 \%(20-66)$ \\
Consensus & $55 \%(27-73)$ & $100 \%(63-100)$ & $100 \%(69-100)$ & $47 \%(21-69)$ \\
\hline
\end{tabular}

Note:-PPV indicates positive predictive value; NPV, negative predictive value. were respectively 85\% (95\% CI, 62-97), 100\% (95\% CI, 63-100), $100 \%$ (95\% CI, 80-100), and 73\% (95\% CI, 39-94). Individual results are described in Table 5. All residual BAVMs detected on ASL/TOF were confirmed on DSA. However, in 3 patients, while ASL/TOF findings were interpreted as negative, residual AVS was demonstrated on DSA. Of those 3 patients, imaging of 1 patient demonstrated faint hypersignal on ASL images at the site of the nidus, considered suggestive of a residual BAVM by all 3 readers; however, the lack of signal abnormality on anatomic MR images prevented the readers, who were blinded to the BAVM location, from detecting the nidus site (Fig 4). Comparison of TOF and ASL ratings showed that all residual BAVMs and nidus obliterations detected on TOF images were correctly classified on ASL images. In 3 patients with negative findings on TOF images, ASL showed the presence of a residual AVS in 2 patients and a $5-\mathrm{mm}$ residual BAVM in 1 patient (Fig 2).

\section{Comparison of Contrast- Enhanced MR Imaging Sequences and ASL/TOF}

ASL/TOF demonstrated superior residual BAVM detection compared with contrast-enhanced MR imaging $(P=.008)$. In 5 patients with AVS and in 1 with a $5-\mathrm{mm}$ nidus, all shown on DSA, a residual BAVM was only

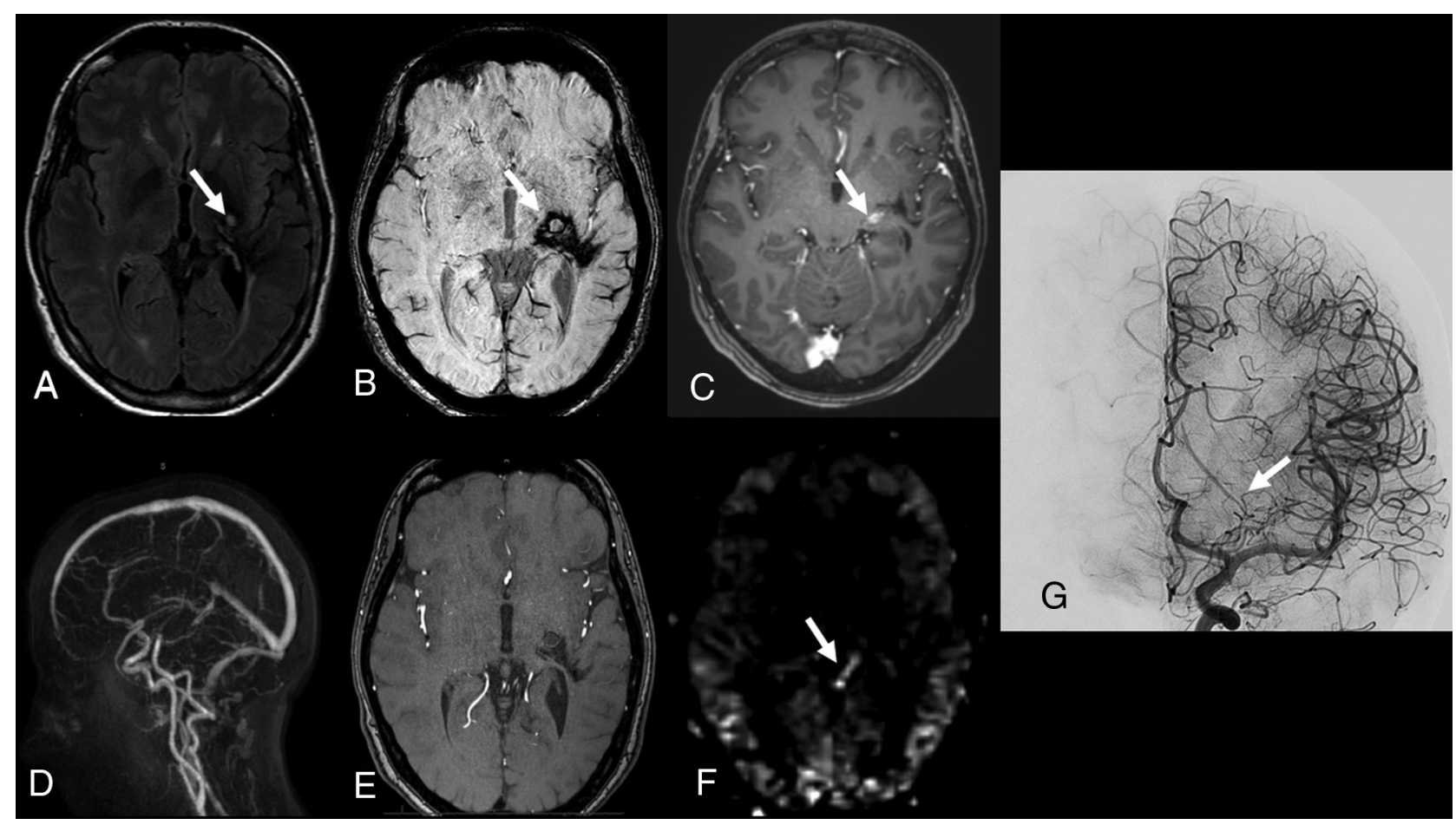

FIG 2. Patient 2. Follow-up MR imaging 7 years after radiosurgery for a ruptured left thalamic brain AVM. A, Axial FLAIR shows slight hypersignal at the site of the radiosurgically treated nidus (arrow). B, SWI shows hypointense hemorrhagic focus in the left deep nuclei (arrow). C, Postcontrast 3D TI GRE axial image shows nodular contrast enhancement at the nidus site (arrow) due to radio-induced changes. $D$, Sagittal view from 4D contrast MRA does not show residual BAVM. Source image from 3D-TOF (E) does not demonstrate abnormal signal intensity at the nidus site, whereas the ASL image $(F)$ shows focal tubular hyperintense signal of the deep draining vein (arrow). G, DSA with an anteroposterior intracranial view from a left carotid angiogram confirms the presence of residual AVS without a measurable nidus. Only early draining vein contrast filling is detected on an angiogram (arrow). 


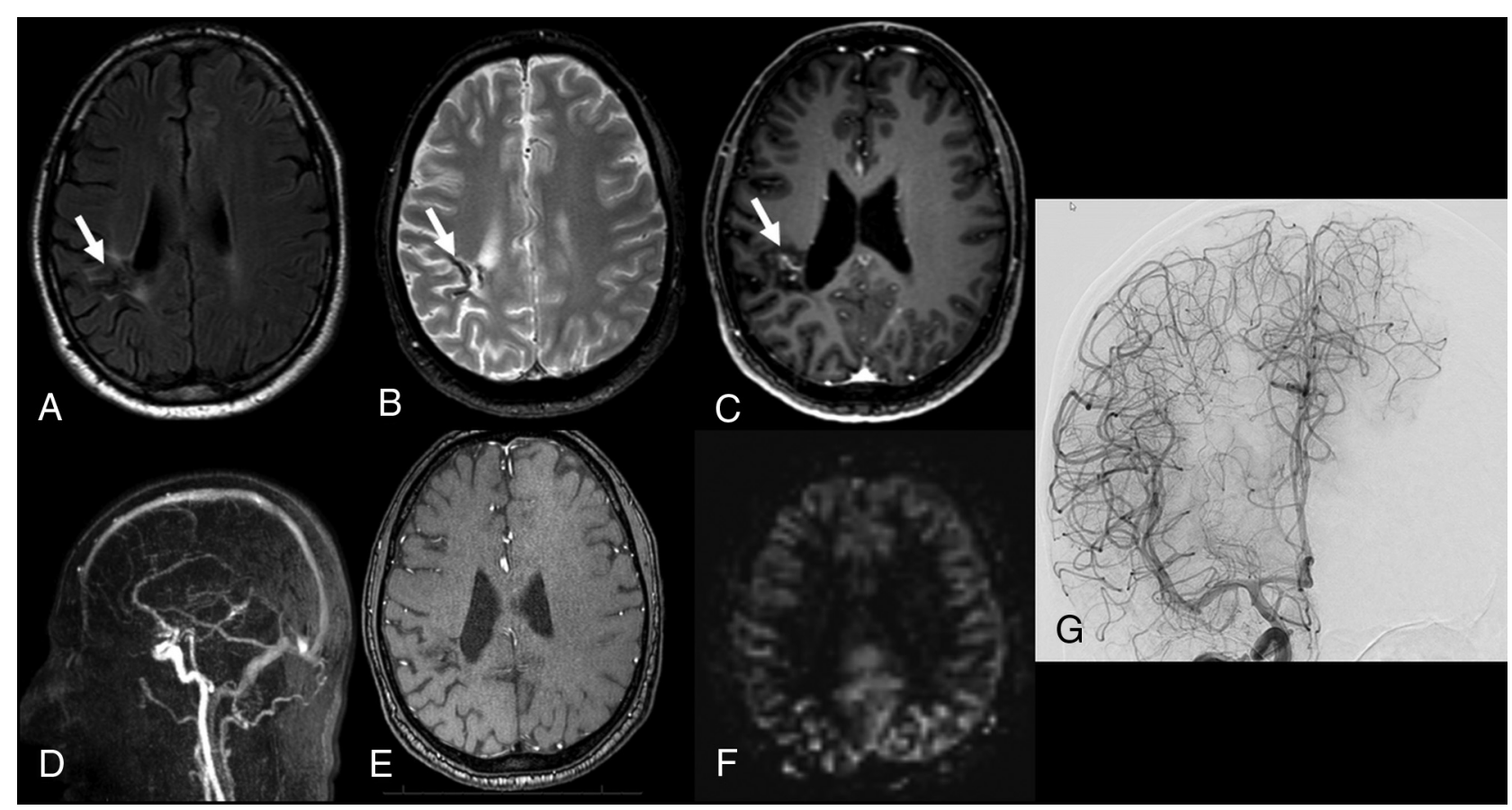

FIG 3. Patient 22. Follow-up MR imaging 4 years after radiosurgery for a ruptured right parietal brain AVM. A, Axial FLAIR shows mixed signal at the site of a radiosurgically treated BAVM (arrow). B, Axial T2* shows subcortical right parietal hypointense signal related to prior hemorrhage (arrow). C, Postcontrast 3D TI GRE shows linear-like heterogeneous contrast enhancement at the nidus site (arrow) due to radiation injury misinterpreted as a residual BAVM by 2 readers. 4D contrast MRA $(D)$, source image from 3D TOF $(E)$, and ASL $(F)$ do not reveal a residual BAVM. $G$, DSA with an anteroposterior intracranial view from a right carotid angiogram confirms the absence of a residual BAVM.

Table 5: Sensitivity, specificity, PPV, and NPV of the second sitting (FLAIR, T2*, and ASL/TOF images)

\begin{tabular}{lcccr}
\hline \multicolumn{1}{c}{ Readers } & Sensitivity (95\% CI) & Specificity (95\% CI) & PPV (95\% CI) & NPV (95\% CI) \\
\hline 1 & $85 \%(62-97)$ & $100 \%(63-100)$ & $100 \%(80-100)$ & $72.7 \%(39-94)$ \\
2 & $85 \%(62-97)$ & $94.4 \%(47-100)$ & $87.5 \%(72-100)$ & $70 \%(35-93)$ \\
3 & $80 \%(56-94)$ & $94.1 \%(47-100)$ & $87.5 \%(71-100)$ & $63.6 \%(31-89)$ \\
Consensus & $85 \%(62-97)$ & $100 \%(63-100)$ & $100 \%(80-100)$ & $73 \%(39-94)$ \\
\hline
\end{tabular}

Note:-PPV indicates positive predictive value; NPV, negative predictive value.

detected on ASL/TOF, while the nidus was considered obliterated on contrast-enhanced MR images (Fig 5). Of the 5 patients with DSA-proved AVS considered occluded on contrast-enhanced MR imaging, ASL showed a residual BAVM in 3. Nine patients were given an uncertain score (3/5) at the first session (contrast-enhanced MR imaging), and 6, patients at the third session (all MR images). With ASL/TOF alone, no uncertain score was given (Fig 6).

\section{DISCUSSION}

In this study, noncontrast 3T MR imaging using ASL and 3DTOF was found to be more sensitive than contrast-enhanced MR imaging for the detection of a residual nidus in patients with small $(<20 \mathrm{~mm})$ BAVMs treated by radiosurgery. We found that the clinical value of noncontrast MR images was even better when they were analyzed without postcontrast 3D T1 GRE images, in part owing to confusion between radiation-induced enhancement and residual BAVMs.

To our knowledge, ours is the first study to evaluate the comparative performance of noncontrast MR imaging and contrast-enhanced MR imaging for the follow-up of patients treated radiosurgically for a small BAVM. ASL and TOF sequences were interpreted conjointly, but the separate ratings of ASL and TOF showed the potential benefit of ASL compared with TOF for detecting a residual BAVM after radiosurgery because a residual DSA-proved BAVM was only detected on ASL images in 3 patients. A small number of published studies, to date, have discussed the usefulness of ASL in the evaluation of intracranial arteriovenous dural fistulas and/or BAVMs, ${ }^{17,20-23}$ including 2 studies of radiosurgically treated patients with BAVMs. ${ }^{21,23}$ In our study, only patients with small $(<20 \mathrm{~mm})$ BAVMs treated by radiosurgery were included. In this highly selective cohort, and although follow-up angiography demonstrated the most evidence of either nidus obliteration or subtle residual AVS, we showed that detection of residual shunting with ASL/TOF was possible with excellent interobserver agreement and superior sensitivity in comparison with contrastenhanced MR imaging, with a high confidence level and a zero rate of uncertain scores to detect a residual flow on ASL/TOF images.

When the PLD is appropriately obtained, ASL images of a healthy brain do not show any hypersignal in the venous structures because the transit time of labeled arterial protons in the capillary bed is longer than the PLD. Conversely, when AVS is present, rapid passage of labeled protons into the venous compartment results in hypersignal in the nidus and/or the draining 


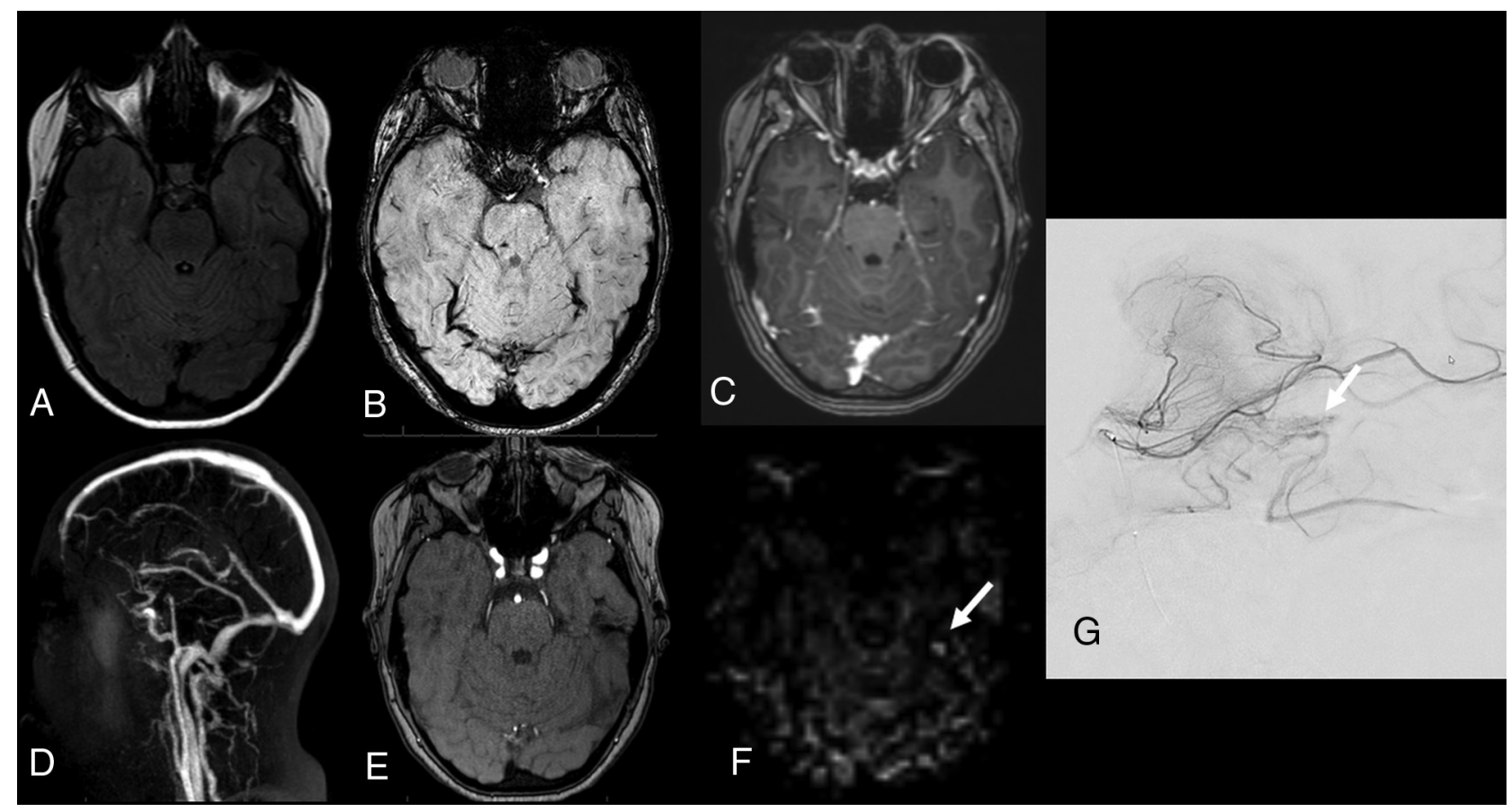

FIG 4. Patient 9 . Follow-up MR imaging 6 years after radiosurgery for an unruptured left posterior temporal brain AVM. Tl-weighted noncontrast $(A)$, SWI (B), postcontrast 3D TI GRE (C), 4D contrast-MRA (D), and a source image from 3D-TOF (E) do not show signal abnormality. F, ASL shows faint hyperintense signal (arrow) not interpreted as a residual BAVM by the 3 readers who were blinded to the BAVM location. G, DSA with a sagittal intracranial view from a superselective left temporal artery angiogram shows an early superficial draining vein contrast filling (arrow).

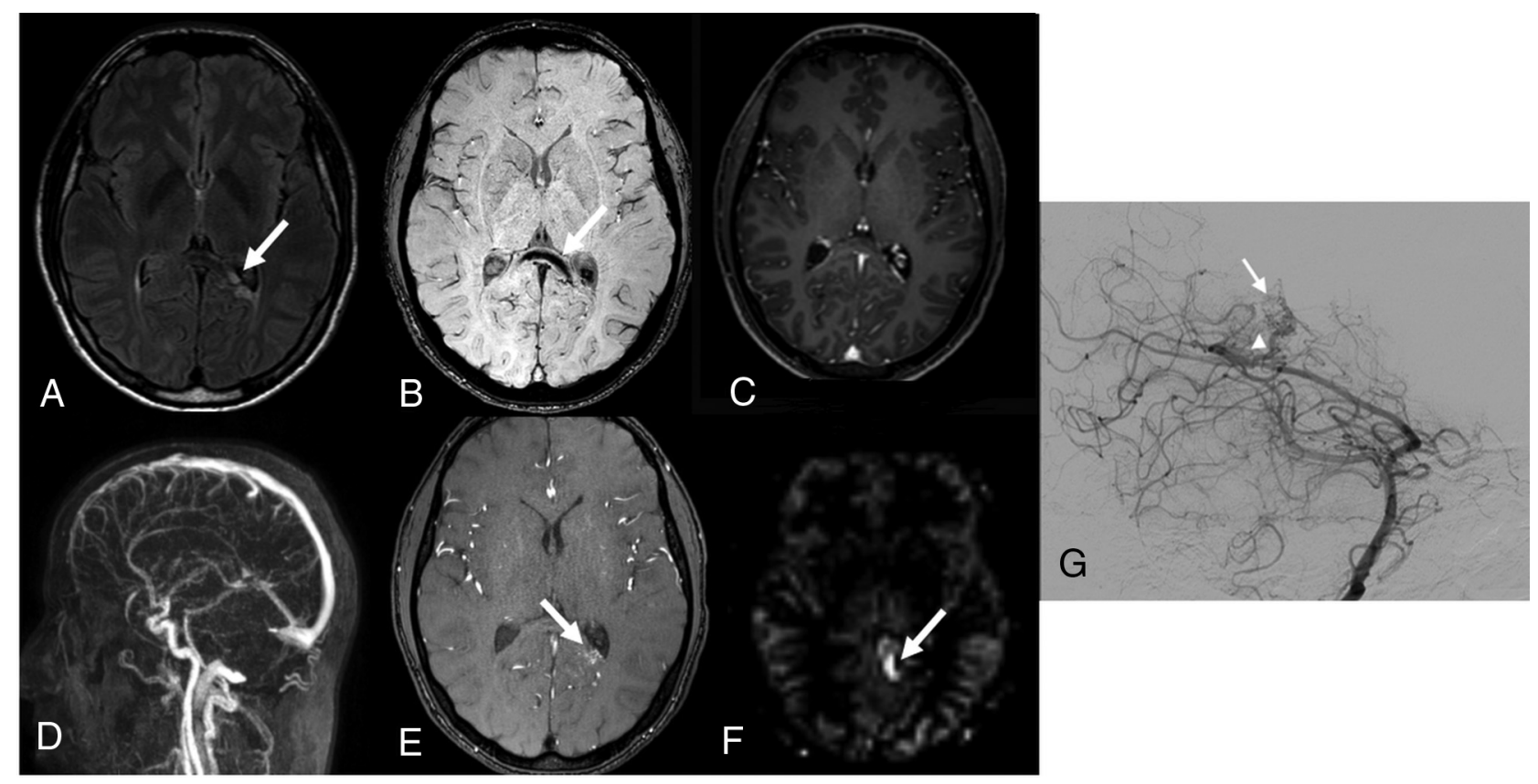

FIG 5. Patient 21. Follow-up MR imaging 2 years after radiosurgery for a ruptured parietal brain AVM adjacent to the corpus callosum. A, Axial FLAIR shows slight hypersignal at the nidus site (arrow). B, T2* image shows hypointense prior hemorrhagic focus in the corpus callosum (arrow). Postcontrast 3D TI GRE (C) and 4D contrast MRA (D) do not reveal a residual BAVM. E, Source image from 3D-TOF shows a residual 5-mm nidus (arrow). F, ASL image shows focal hyperintense signal of a deep draining vein (arrow). G, DSA, oblique intracranial view from a left vertebral angiogram confirms the presence of a residual nidus (arrow) with an early deep draining vein (arrowhead).

veins. In a study published in 2017 , Kodera et $\mathrm{al},{ }^{23}$ evaluating 7 patients with BAVMs treated by radiosurgery, showed, in all 7 patients, a correlation between BAVM obliteration on DSA and lack of BAVM detection on ASL images. More recently, Heit et $\mathrm{al}^{21}$ in a retrospective study including 15 patients with larger BAVM nidus sizes in comparison with our cohort, showed $100 \%$ sensitivity and 95\% specificity of ASL for the detection of residual BAVMs after radiosurgery. These data suggest a role for ASL in 


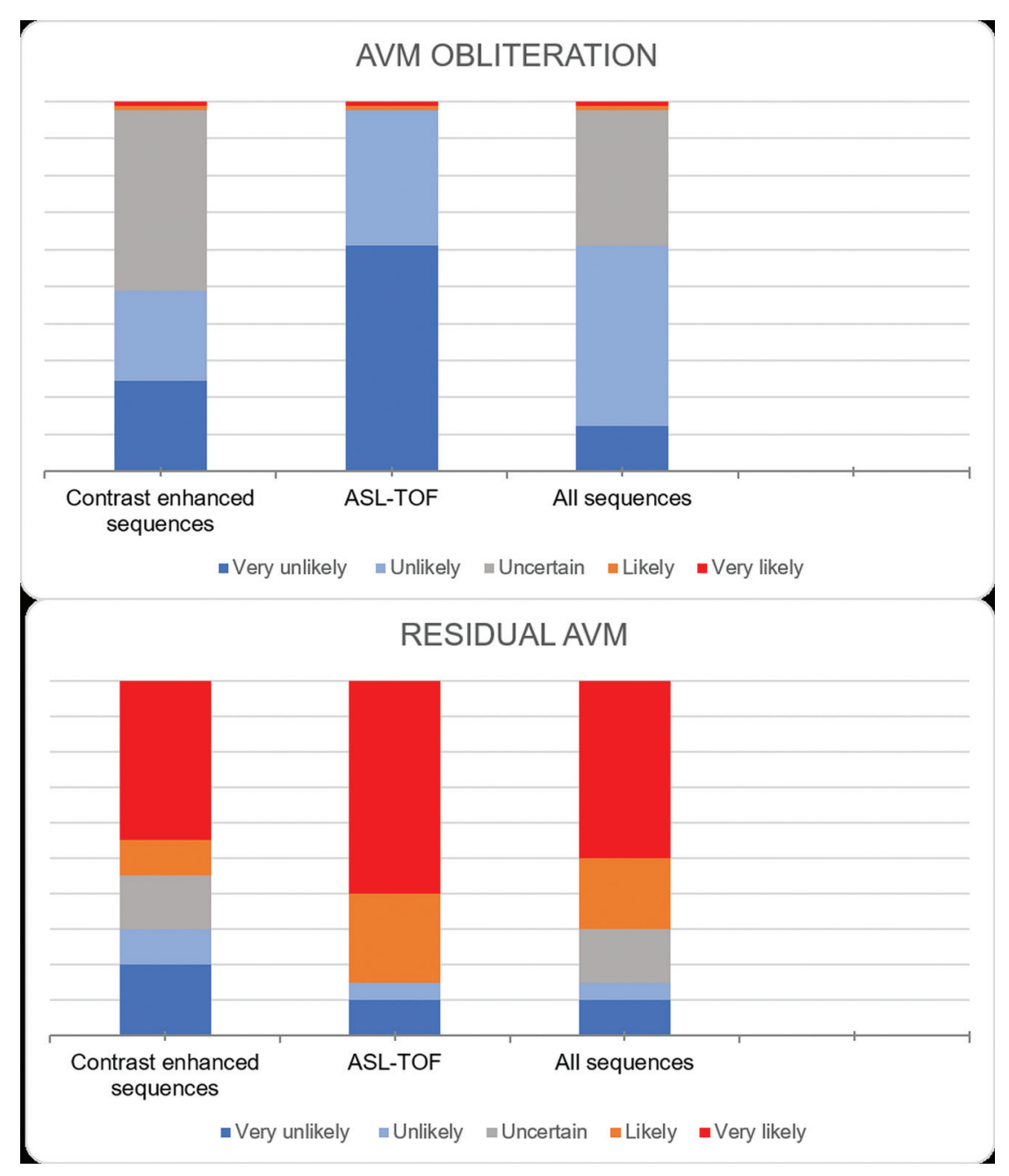

FIG 6. Distribution of the 5-point scale of the 3 sittings in the 28 patients with and without residual brain AVMs on DSA. In the "AVM OBLITERATION" group, reader accuracy and confidence increased with ASL/TOF with zero uncertain scores and more scores of very unlikely. In the "RESIDUAL AVM" group, reader accuracy and confidence increased with ASL/TOF with zero uncertain scores and more scores of very likely.

3T MR imaging protocols to determine the adequate timing for follow-up DSA in treated patients.

In addition, although previous studies have demonstrated the usefulness of $3 \mathrm{D}$-TOF in detecting intracranial arteriovenous shunting or in characterizing BAVMs, ${ }^{13}$ ours is the first study to combine TOF-MRA with ASL for the follow-up of radiosurgically treated patients with BAVMs. Our study also suggests a high interobserver confidence level in detecting residual shunting using combined sequences as opposed to contrast-enhanced MR imaging alone, even though ASL appears to show the highest sensitivity in residual nidal flow detection. The reproducibility of our results may be limited because the spatial resolution of TOF and ASL sequences was optimized in this study by the use of a 3T magnet and 32-channel coil.

The sensitivity of $4 \mathrm{D}$ contrast MRA for the detection of residual BAVMs in the present study was lower compared with that reported in a previous study by Soize et $\mathrm{al},{ }^{10}$ who followed up a cohort of patients treated by embolization, radiosurgery, and/or surgery. They showed an overall sensitivity of $73 \%$ for the detection of residual BAVMs compared with $55 \%$ in the present study. This discrepancy may be explained by the selected patient group in our study, because 8 of the 28 BAVMs were obliterated and 8 had only residual AVS without a measurable nidus.

On postcontrast 3D T1 GRE images, various aspects of radiation-induced contrast enhancement were found in this study that might lead to confusion between a residual BAVM or radiation-induced changes, translating into scores of uncertain in some patients. These radiation-induced imaging changes may be related to various factors, including blood-brain barrier damage due to inflammation, dilated capillary vessels, or neovascularization in the wall of a perinidal cavity with neoangiogenetic nodule formation. $^{24}$ Negative findings on $4 \mathrm{D}$ contrast MRA did not provide additional information because they could be related to either radiationinduced imaging changes or a residual BAVM, explained by the limited temporal and spatial resolution inherent to the $4 \mathrm{D}$ contrast MRA technique, which hindered the detection of small nidi with decreased flow.

Most important, these preliminary data also suggest that for long-term follow-up of patients with BAVMs treated by radiosurgery, the use of a gadolinium contrast medium may not be systematically required; this possibility could reduce the likelihood of long-term effects of gadolinium retention, including within deep nuclei. $^{11}$

Several limitations may be highlighted in our study. First, this is a retrospective, single-center study design and, therefore, subject to a potential selection bias. Prospective randomized studies with properly selected control groups would be useful to support our preliminary data. Second, the small sample size may not easily allow reproducibility, especially considering the low prevalence of small BAVMs. Third, the lack of a control group of patients without BAVMs, who would have been evaluated with both DSA and MR imaging with ASL/TOF, may reduce the statistical power; however, the performance of DSA and the administration of gadolinium contrast in healthy subjects may not have been ethically justifiable. Last, 2D-ASL with a single PLD value was the technique used in this study; there have been recent technical advances with commercially available $3 \mathrm{D}$-ASL sequences, including multi-PLD, that may potentially improve the image quality and robustness of image interpretation. ${ }^{25}$ Despite these limitations, we believe that our results suggest 
that as advanced MR imaging techniques such as ASL become generalized, the systematic use of contrast-enhanced MR images may become unwarranted in clinical routine to guide decisionmaking about the timing of appropriate DSA follow-up in this group of patients.

\section{CONCLUSIONS}

In this study, contrast-enhanced MR images were found to be inferior to noncontrast MR imaging, including TOF and ASL at 3T, in demonstrating a residual nidus for the follow-up of patients with BAVMs treated by radiosurgery. These data suggest that ASL/TOF could be used to decide the appropriate timing for follow-up DSA to confirm AVM obliteration. As a result, sequential injections of gadolinium contrast medium could be avoided, sparing patients the potential risk of gadolinium accumulation, including within deep brain nuclei. Further studies including larger groups of patients are needed to confirm this hypothesis.

Disclosures: Nicolas Bricout-RELATED: Consulting Fee or Honorarium: None.

\section{REFERENCES}

1. Arteriovenous Malformation Study Group. Arteriovenous malformations of the brain in adults. N Engl J Med 1999;340:1812-18 CrossRef Medline

2. Laakso A, Hernesniemi J. Arteriovenous malformations: epidemiology and clinical presentation. Neurosurg Clin N Am 2012;23:1-6 CrossRef Medline

3. Yamada S, Takagi Y, Nozaki K, et al. Risk factors for subsequent hemorrhage in patients with cerebral arteriovenous malformations. J Neurosurg 2007;107:965-72 CrossRef Medline

4. van Beijnum J, van der Worp HB, Buis DR, et al. Treatment of brain arteriovenous malformations: a systematic review and meta-analysis. JAMA 2011;306:2011-19 CrossRef Medline

5. Vlaskou Badra E, Ermiş E, Mordasini P, et al. Radiosurgery and radiotherapy for arteriovenous malformations: outcome predictors and review of the literature. J Neurosurg Sci 2018;62:490-504 CrossRef Medline

6. Friedman WA, Bova FJ. Radiosurgery for arteriovenous malformations. Neurol Res 2011;33:803-19 CrossRef Medline

7. Chowdhury AH, Ghose SK, Mohammad QD, et al. Digital subtraction angiography is superior to magnetic resonance angiography in diagnosis of cerebral arteriovenous malformation. Mymensingh Med J MMJ 2015;24:356-65 Medline

8. Willinsky RA, Taylor SM, terBrugge K, et al. Neurologic complications of cerebral angiography: prospective analysis of 2,899 procedures and review of the literature. Radiology 2003;227:522-28 CrossRef Medline

9. Hadizadeh DR, von Falkenhausen M, Gieseke J, et al. Cerebral arteriovenous malformation: Spetzler-Martin classification at subsecond-temporal-resolution four-dimensional MR angiography compared with that at DSA. Radiology 2008;246:205-13 CrossRef Medline
10. Soize S, Bouquigny F, Kadziolka K, et al. Value of 4D MR angiography at 3T compared with DSA for the follow-up of treated brain arteriovenous malformation. AJNR Am J Neuroradiol 2014;35: 1903-09 CrossRef Medline

11. Choi JW, Moon WJ. Gadolinium deposition in the brain: current updates. Korean J Radiology 2019;20:134-47 CrossRef Medline

12. Adin ME, Kleinberg L, Vaidya D, et al. Hyperintense dentate nuclei on T1-weighted MRI: relation to repeat gadolinium administration. AJNR Am J Neuroradiol 2015;36:1859-65 CrossRef Medline

13. Buis DR, Bot JC, Barkhof F, et al. The predictive value of $3 D$ time-of-flight MR angiography in assessment of brain arteriovenous malformation obliteration after radiosurgery. AJNR Am J Neuroradiol 2012;33:232-38 CrossRef Medline

14. Grade M, Hernandez Tamames JA, Pizzini FB, et al. A neuroradiologist's guide to arterial spin labeling MRI in clinical practice. Neuroradiology 2015;57:1181-202 CrossRef Medline

15. Haller S, Zaharchuk G, Thomas DL, et al. Arterial spin labeling perfusion of the brain: emerging clinical applications. Radiology 2016;281:337-56 CrossRef Medline

16. Wolf RL, Wang J, Detre JA, et al. Arteriovenous shunt visualization in arteriovenous malformations with arterial spin-labeling MR imaging. AJNR Am J Neuroradiol 2008;29:681-87 CrossRef Medline

17. Le TT, Fischbein NJ, André JB, et al. Identification of venous signal on arterial spin labeling improves diagnosis of dural arteriovenous fistulas and small arteriovenous malformations. AJNR Am J Neuroradiol 2012;33:61-68 CrossRef Medline

18. Cohen J. A coefficient of agreement for nominal scales. Educ Psychol Meas 1960;20:37-46 CrossRef

19. Fleiss JL. Reliability of measurement. In: Fleiss JL. The Design and Analysis of Clinical Experiments. New York: John Wiley \& Sons; 1986:1-32

20. Amukotuwa SA, Marks MP, Zaharchuk G, et al. Arterial spin-labeling improves detection of intracranial dural arteriovenous fistulas with MRI. AJNR Am J Neuroradiol 2018;39:669-77 CrossRef Medline

21. Heit JJ, Thakur NH, Iv M, et al. Arterial-spin labeling MRI identifies residual cerebral arteriovenous malformation following stereotactic radiosurgery treatment. J Neuroradiol 2019 Jan 15. [Epub ahead of print] CrossRef Medline

22. Hodel J, Leclerc X, Kalsoum E, et al. Intracranial arteriovenous shunting: detection with arterial spin-labeling and susceptibilityweighted imaging combined. AJNR Am J Neuroradiol 2017;38:71-76 CrossRef Medline

23. Kodera T, Arai Y, Arishima H, et al. Evaluation of obliteration of arteriovenous malformations after stereotactic radiosurgery with arterial spin labeling MR imaging. Br J Neurosurg 2017;31:641-47 CrossRef Medline

24. Malikova H, Koubska E, Vojtech Z, et al. Late morphological changes after radiosurgery of brain arteriovenous malformations: an MRI study. Acta Neurochir (Wien) 2016;158:1683-90 CrossRef Medline

25. Johnston ME, Lu K, Maldjian JA, et al. Multi-TI arterial spin labeling MRI with variable TR and bolus duration for cerebral blood flow and arterial transit time mapping. IEEE Trans Med Imaging 2015;34:1392-402 CrossRef Medline 